\title{
CKAP4 is identified as a receptor for Dickkopf in cancer cells
}

\author{
Dheeraj Bhavanasi,, Kelsey F. Speer, ${ }^{2}$ and Peter S. Klein ${ }^{1,2}$ \\ 'Department of Medicine, (Hematology-Oncology), and ²Cell and Molecular Biology Graduate Group, Perelman School of Medicine at the University of Pennsylvania, Philadelphia, Pennsylvania, USA.
}

\begin{abstract}
The secretory protein Dickkopf-1 (DKK-1) is a known Wnt antagonist and has been shown to suppress tumorigenesis in some cancer cells; however, it is also upregulated in many types of cancer and associated with poor prognosis. Wnt-independent mechanisms by which DKK-1 promotes cancer cell proliferation are not well understood. In this issue of the $J C I$, Kimura and colleagues demonstrate that DKK-1 interacts with cytoskeletonassociated protein 4 (CKAP4) to promote activation of AKT. They show that both DKK-1 and CKAP4 are frequently upregulated in pancreatic and lung cancers. Importantly, targeting this interaction with an anti-CKAP4 antibody prevented tumor formation in murine xenograft models. These results identify a previously unrecognized DKK-1-mediated pathway and suggest CKAP4 as a potential therapeutic target for certain cancers.
\end{abstract}

\section{Divergent functions for Dickkopf-1}

Dickkopf-1 (DKK-1) was first identified in Xenopus as an extracellular antagonist of canonical Wnt signaling (1), a fundamental pathway that controls cell fate, survival, and proliferation. Aberrant activation of Wnt signaling also drives tumorigenesis in colon, breast, hepatocellular, and many other cancers (2-4). As a Wnt signaling inhibitor, DKK-1 suppresses tumorigenesis in some cancer cells, including renal cell carcinoma (5), breast carcinoma (6), and colon cancer cells (7-9). However, DKK-1 is often elevated in cancers, is associated with aggressive disease, and promotes proliferation in multiple cell types $(10,11)$, suggesting that DKK-1 also functions independently of Wnt inhibition.

In this issue, Kimura and colleagues investigate the molecular events that lead to DKK-1-induced cancer cell proliferation (12). Their work has identified a Wnt-independent signaling role for DKK-1 through interaction with the type-II transmembrane protein cytoskeleton-associated protein 4 (CKAP4; also known as p63, ERGIC-63, or CLIMP-63). These findings also indicate that the DKK-1/CKAP4 interaction represents a promising therapeutic target for cancer.

\section{CKAP4 is a DKK-1 receptor that activates AKT signaling}

DKK family proteins contain two cysteinerich domains (CRDs). The C-terminal CRD (CRD-2) of DKK-1 inhibits Wnt signaling by directly binding to the Wnt coreceptors LRP5/6 (LDL-related proteins 5 and 6), thereby preventing interaction with Wnts, and by binding to the transmembrane protein KREMEN to promote LRP5/6 internalization (11, 13-15). Kimura et al. have now demonstrated that CRD-1 of DKK-1 binds to CKAP4 at the apical surface of epithelial cells (Figure 1 and ref. 12). Furthermore, DKK-1 binds to CKAP4 with high affinity that is comparable to but independent of its interaction with LRP5/6. Upon DKK-1 binding, CKAP4 recruits phosphatidylinositol-3 kinase (PI3K) to its cytoplasmic domain through interaction with the SH3 domain of the p85 $\alpha$ subunit of PI3K. Through this mechanism, DKK-1 activates AKT and promotes proliferation. These findings substantially expand our understanding of DKK-1 signaling mechanisms and define a previously unrecognized input to the PI3K/AKT pathway.

The findings of Kimura et al. also raise questions about the potential functions of other DKK family members (12). The DKK family comprises four conserved proteins, DKK-1 through -4 (and DKK-like 1, which lacks the CRDs). DKK-1 antagonizes Wnt $\beta$-catenin signaling, activates AKT (as shown here), and may also activate JNK signaling (16); however, the functions of other DKK family members are less well characterized. DKK-2 and -4 antagonize Wnt signaling, similar to DKK-1, but DKK-2 can also activate canonical Wnt signaling depending on the cellular context $(11,17)$. In contrast, DKK-3 positively regulates Wnt signaling (18) and binds to integrin $\alpha 6 \mathrm{~b}$ to induce myogenesis in zebrafish (19). As Kimura and colleagues point out (12), CRD-1 is highly conserved among the four $D k k$ genes, raising the possibility that DKK-2, -3 , and -4 could also interact with CKAP4. In support of this proposal, DKK-3 protects cells from proapoptotic stimuli (18). While the mechanism of this DKK-3-mediated effect has not been characterized, the prosurvival function of DKK-3 could be mediated by interaction with CKAP4 and subsequent AKT activation.

\section{Targeting DKK-1/CKAP4 interactions in cancer}

$D K K 1$ is a Wnt target gene and functions in a negative feedback loop that may in some cases suppress tumorigenesis. Consistent with a tumor suppressor function, $D K K$ family genes are silenced by hypermethylation in colon cancer, renal cell carcinoma, and melanoma. However, DKK-1 expression is increased in many other cancers, including multiple myeloma, hepatocellular carcinoma, and ovarian adeno- 


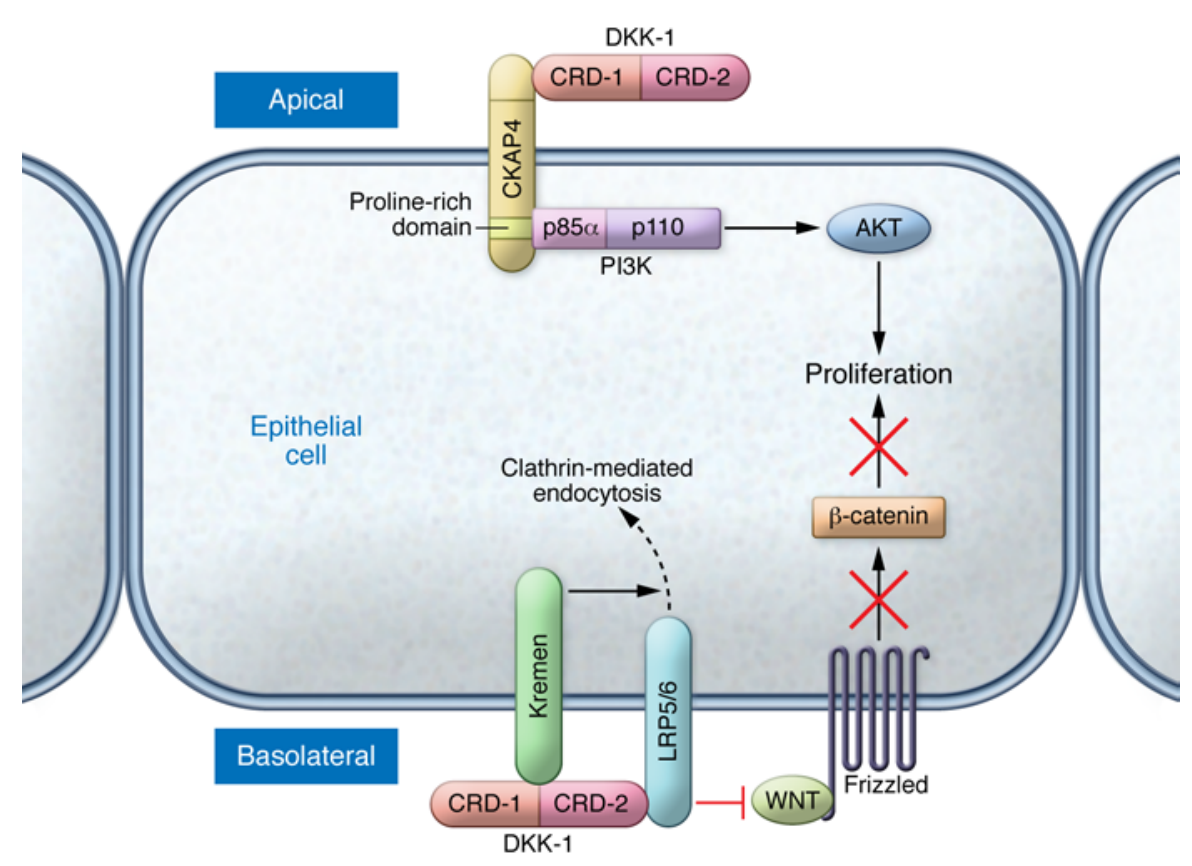

carcinoma and is associated with more aggressive behavior (11). In these cases, the findings of Kimura et al. suggest a second, potentially counteracting effect of DKK-1 to stimulate proliferation through an AKT-dependent, Wnt-independent mechanism (12).

Thus, the DKK-1/CKAP4 interaction could be a promising therapeutic target for some cancers. Kimura and colleagues found that approximately $70 \%$ of pancreatic ductal adenocarcinomas, lung adenocarcinomas, and lung squamous cell carcinomas express high levels of DKK-1 and CKAP4 compared with adjacent, nontransformed tissue and that expression of both proteins correlates with lower relapse-free survival (12). Kimura et al. also demonstrated that DKK-1-expressing cancer cells require CKAP4 expression for tumor growth in mouse xenograft models. Furthermore, an antibody targeting the extracellular domain of CKAP4 suppressed tumorigenesis in DKK-1-expressing cell lines and in a mouse xenograft model. These findings are consistent with published data showing that anti-DKK-1 antibodies impair growth of engrafted human cancers, such as multiple myeloma, in mice $(10,20,21)$. However, given that DKK-1 may also suppress tumors by inhibiting Wnt signaling, an antibody to CKAP4 may have more specific, antiproliferative effects that spare the potential tumor suppressor function of DKK-1. Therefore, this
CKAP4-targeting antibody may be a valuable therapeutic agent for DKK-1/CKAP4positive cancers.

\section{Conclusions and future directions}

This study by Kimura and colleagues reveals a previously unknown signaling pathway in which DKK-1 binds to CKAP4, resulting in activation of $\mathrm{AKT}$ through PI3K to enhance Wnt-independent cellular proliferation (12). The thorough signaling data presented by Kimura et al. provide an explanation for the proliferative effects of DKK-1 and the strong correlation between elevated DKK-1 and aggressive tumor behavior. This work also provides intriguing preclinical data to support the use of anti-CKAP4 antibodies in DKK-1/ CKAP4-positive tumors.

DKK-1 is often used experimentally to test whether a biological process is mediated by canonical Wnt signaling. The findings of Kimura et al., along with other studies supporting non-Wnt functions for DKK-1 $(11,16,19,22)$, underscore the limitations of this approach by showing that DKK-1 is not a specific inhibitor of canonical Wnt signaling. Kimura et al. also raise questions for further study. It will be important to determine whether DKK-1 binds directly to CKAP4 or through an intermediate binding partner. Additionally, interaction of CKAP4 with DKK-2 through -4, which contain the highly conserved CRD-1,
Figure 1. DKK-1 stimulates proliferation by binding to CKAP4 and activating AKT. DKK-1 is secreted from epithelial cells and binds to the extracellular domain of CKAP4 at the apical surface of the cell. The CRD-1 of DKK-1 and the leucine zipper (LZ) domain of CKAP4 are required for this interaction (although a direct interaction between the two has not yet been shown). Upon DKK-1 binding, CKAP4 binds to the $p 85 \alpha$ subunit of PI3K to activate PI3K/AKT signaling and stimulate cancer cell proliferation. This signaling motif is in contrast to the well-established role for DKK-1 as a canonical Wnt antagonist, in which CRD-2 of DKK-1 binds to the Wnt coreceptor LRP5/6 (preventing interaction with Wnts) and to Kremen (driving internalization of LRP5/6).

also remains to be investigated. Other questions to be addressed include whether expression of CKAP 4 and DKK-1 will play a role in diagnosis or staging of pancreatic or lung cancers and whether these observations will extend to other cancers. Finally, the comprehensive mechanistic experiments in this study focused on cell lines and xenograft models, leaving open the question of whether the DKK-1/CKAP4 interaction plays a role in primary tumor proliferation or in the proliferation of nontransformed cells. Kimura and colleagues did not observe any obvious effect on normal tissues in their mouse model after 4 weeks of anti-CKAP4 antibody treatment, which is reassuring that inhibiting DKK-1 does not have short-term deleterious effects on normal proliferative tissues; however, the longer-term effects of this strategy are not yet known. Nevertheless, the identification of a signaling mechanism for DKK-1 and the strong preclinical observations presented here are sure to lead to exciting work in the future.

\section{Acknowledgments}

This work is supported by grants from the NIH (5R01MH100923 and 7R01GM107435), the Institute for Translational Medicine and Applied Therapeutics, the Institute for Regenerative Medicine, and the Orphan Disease Center at the University of Pennsylvania School of Medicine. 
Address correspondence to: Peter S. Klein, 9-103 SCTR, 3400 Civic Center Blvd., Philadelphia, Pennsylvania 19104, USA. Phone: 215.898.2179; E-mail:pklein@upenn.edu.

1. Glinka A, Wu W, Delius H, Monaghan AP, Blumenstock C, Niehrs C. Dickkopf-1 is a member of a new family of secreted proteins and functions in head induction. Nature. 1998;391(6665):357-362.

2. Reya T, Clevers H. Wnt signalling in stem cells and cancer. Nature. 2005;434(7035):843-850.

3. Anastas JN, Moon RT. WNT signalling pathways as therapeutic targets in cancer. Nat Rev Cancer. 2013;13(1):11-26.

4. Polakis P. Wnt signaling and cancer. Genes Dev. 2000;14(15):1837-1851.

5. Hirata $\mathrm{H}$, et al. Wnt antagonist DKK1 acts as a tumor suppressor gene that induces apoptosis and inhibits proliferation in human renal cell carcinoma. Int J Cancer. 2011;128(8):1793-1803.

6. Mikheev AM, et al. Dickkopf-1 mediated tumor suppression in human breast carcinoma cells. Breast Cancer Res Treat. 2008;112(2):263-273.

7. González-Sancho JM, et al. The Wnt antagonist DICKKOPF-1 gene is a downstream target of $\beta$-catenin/TCF and is downregulated in human colon cancer. Oncogene.
2005;24(6):1098-1103.

8. Zhu Y, et al. Human mesenchymal stem cells inhibit cancer cell proliferation by secreting DKK-1. Leukemia. 2009;23(5):925-933.

9. Qiao L, Xu ZL, Zhao TJ, Ye LH, Zhang XD. Dkk-1 secreted by mesenchymal stem cells inhibits growth of breast cancer cells via depression of Wnt signalling. Cancer Lett. 2008;269(1):67-77.

10. Sato N, et al. Wnt inhibitor Dickkopf-1 as a target for passive cancer immunotherapy. Cancer Res. 2010;70(13):5326-5336.

11. Niehrs C. Function and biological roles of the Dickkopf family of Wnt modulators. Oncogene. 2006;25(57):7469-7481.

12. Kimura H, et al. CKAP4 is a Dickkopf1 receptor and is involved in tumor progression. JClin Invest. 2016;126(7):2689-2705.

13. Bafico A, Liu G, Yaniv A, Gazit A, Aaronson SA. Novel mechanism of Wnt signalling inhibition mediated by Dickkopf-1 interaction with LRP6/ Arrow. Nat Cell Biol. 2001;3(7):683-686.

14. Mao B, et al. LDL-receptor-related protein 6 is a receptor for Dickkopf proteins. Nature. 2001;411(6835):321-325.

15. Semënov MV, Tamai K, Brott BK, Kühl M, Sokol S, He X. Head inducer Dickkopf-1 is a ligand for Wnt coreceptor LRP6. Curr Biol. 2001;11(12):951-961.
16. Lee AY, et al. Dickkopf-1 antagonizes Wnt signaling independent of beta-catenin in human mesothelioma. Biochem Biophys Res Commun. 2004;323(4):1246-1250.

17. Mao B, Niehrs C. Kremen2 modulates Dickkopf2 activity during Wnt/LRP6 signaling. Gene. 2003;302(1-2):179-183.

18. Nakamura RE, Hunter DD, Yi H, Brunken WJ, Hackam AS. Identification of two novel activities of the Wnt signaling regulator Dickkopf 3 and characterization of its expression in the mouse retina. BMC Cell Biol. 2007;8:52.

19. Fu CY, Su YF, Lee MH, Chang GD, Tsai HJ. Zebrafish Dkk3a protein regulates the activity of myf5 promoter through interaction with membrane receptor integrin $\alpha 6 \mathrm{~b}$. J Biol Chem . 2012;287(47):40031-40042.

20. Pozzi S, et al. In vivo and in vitro effects of a novel anti-Dkk1 neutralizing antibody in multiple myeloma. Bone. 2013;53(2):487-496.

21. Yaccoby S, Ling W, Zhan F, Walker R, Barlogie B, Shaughnessy JD. Antibody-based inhibition of DKK1 suppresses tumor-induced bone resorption and multiple myeloma growth in vivo. Blood. 2007;109(5):2106-2111.

22. Kohn MJ, Kaneko KJ, DePamphilis ML. DkkL1 (Soggy), a Dickkopf family member, localizes to the acrosome during mammalian spermatogenesis. Mol Reprod Dev. 2005;71(4):516-522. 